\title{
REPTILE ADENOVIRUSES IN CATTLE?
}

\author{
B. HARRACH ${ }^{*}$ \\ Veterinary Medical Research Institute, Hungarian Academy of Sciences, \\ H-1581 Budapest, P.O. Box 18, Hungary
}

(Received March 20, 2000; accepted October 17, 2000)

\begin{abstract}
This paper describes a hypothesis on the origin of the members of the recently established adenovirus genus, Atadenovirus, invading cattle, sheep, deer, duck and poultry. Comparison of the phylogenetic trees of adenoviruses and their hosts suggests a very ancient but common origin for the atadenoviruses. The surprisingly large difference between these virus types and other adenoviruses infecting the same host can be easily understood by assuming their separate evolution in different hosts (e.g., in reptiles versus a coevolution with mammals and birds, respectively) followed by a later host switch.
\end{abstract}

Key words: Adenovirus, atadenovirus, evolution, phylogenetics

All adenoviruses studied so far at the molecular level have been isolated either from birds or mammals. Although the presence of adenoviruses has been confirmed in virtually every class of Vertebrata (Clark et al., 1973; Jacobson et al., 1984; Hedrick et al., 1985; Bloch et al., 1986; Davison et al., 1993; Juhasz and Ahne, 1993; Chiocca et al., 1996; Jacobson et al., 1996; Pring-Åkerblom et al., 1997; Lakatos et al., 1999; Lehmkuhl et al., 1999), the official taxonomy distinguishes only two genera (Aviadenovirus and Mastadenovirus), while the less characterized, if at all isolated, adenoviruses of lower vertebrates have no genus attribution (Benkö et al., 2000). The two genera were originally established for the allocation of adenoviruses isolated from birds and mammals. A genus-specific complement binding antigen was also recognized in the members.

Several exceptions, above all adenoviruses with unusual characteristics isolated from cattle - the so-called subgroup 2 bovine adenoviruses (BAdVs) not fitting neatly into their respective genus have been recorded for a long time, but not allocated into a separate taxon (Bartha, 1969; Wigand et al., 1982). A computational molecular biological approach, the phylogenetic analysis of amino acid sequences of selected adenoviral proteins, proved that these viruses (along with an avian adenovirus) do form a third cluster within the family Adenoviridae (Harrach et al., 1997).

The first completely sequenced avian adenovirus genome (FAdV-1 or CELO) demonstrated considerable differences compared to the well-conserved

*E-mail: harrach@vmri.hu; Fax: +36 (1) 467-4076 
organization of mastadenoviruses (Chiocca et al., 1996). The main divergence was found in the early regions, while the majority of the late viral proteins exhibited evident homology to their mammalian counterparts. The full sequence and unique genome organization of an unusual ovine adenovirus isolate (OAV287) has also been published (Vrati et al., 1996). The peculiarities observed in its genome, however, are not characteristic of ovine adenoviruses, the six officially accepted serotypes of which are classical mastadenoviruses (Benkö, 1990; Barbezange et al., 2000). OAV287 is in fact a close relative of the earlier recognized 'irregular' subgroup 2 BAdVs (types 4 through 8 ). Recently, the genome of the egg drop syndrome (EDS) virus has also been completely sequenced (Hess et al., 1997). This virus was also regarded as a deviant member of the Aviadenovirus genus. In spite of the different (avian and mammalian) host origin, the genome organization of the EDS virus and OAV287 is strikingly similar.

Our laboratory has been working on the characterization of adenoviruses of domestic and wild animals including cattle and sheep (Boros et al., 1985; Benkő et al., 1988; Horner et al., 1989; Benkő, 1990; Benkő et al., 1990; Kiss et al., 1996). The phylogenetic analysis of DNA and amino acid sequences of any adenoviral gene suitable for such calculations consistently resulted in the separation of three clusters. Besides the officially accepted Mastadenovirus and Aviadenovirus genera, a third cluster comprising the subgroup 2 BAdVs, OAV287 and the EDS virus always appeared, clearly showing the different evolutionary origin of these viruses (Harrach et al., 1997; Hess et al., 1997; Dán et al., 1998; Harrach and Benkö, 1998; Matiz et al., 1998; Barbezange et al., 2000; Benkő et al., 2000).

The direction of evolution, however, is not evident. One characteristic feature of viruses in this third cluster is the high genomic AT content, therefore the establishment of a new adenovirus genus under the name Atadenovirus has been proposed (Benkö and Harrach, 1998). The overall high AT content easily allows the allocation of regulatory motifs within the coding sequences, thus gene overlaps and short intergenic sequences are present, resulting in a compact genome. Could such a strategy be the result of an evolutionary process, or is it rather an atavistic feature? As an attempt to clarify the question, we have compared the topology of phylogenetic trees based on selected genes of the different adenoviruses and their respective hosts.

\section{Materials and methods}

The FastDNAml calculated phylogenetic tree of the mitochondrial small subunit ribosomal RNA (rRNA) of selected animals was acquired from the Ribosomal Database (Maidak et al., 1997). Since the corresponding sequence from swine was available in the database but not yet included into the phylogenetically 
ordered alignments, the Suggest Tree ('Suggesting a Phylogenetic Placement on the RDP Tree') program was applied to retrieve the desired branch from the existing phylogenetic tree. (The Internet address of this program and the whole Ribosomal Database Project is http://rdpwww.life.uiuc.edu/index2.html.) The TreeView program (available free from http://taxonomy.zoology.gla.ac.uk/rod/ treeview.html) (Page, 1996) was used to visualize the tree obtained in Newick format.

For distance matrix analysis of the amino acid sequence alignment of the adenovirus proteases, the PROTDIST (Dayhoff's PAM 001 scoring matrix) and FITCH (global rearrangements) programs of the PHYLIP package were applied (http://evolution.genetics.washington.edu/phylip.html) (Felsenstein, 1989), and the results were visualized by TreeView, and edited in Word for Windows as described in detail earlier (Harrach and Benkö, 1998).

\section{Results and discussion}

For calculating the phylogenetic distance of the host animals, the mitochondrial small subunit rRNA has been selected (Fig. 1) because its nucleotide sequence is available from the majority of animals described hosting adenoviruses (Russell and Benkö, 1999). Furthermore, a constantly updated collection of the rRNA sequences, their alignment and calculated distances are available through the Internet in the innovative Ribosomal Database that provides freely accessible tools to extract any part of the distance tree and to include not yet aligned sequences (Maidak et al., 1997). However, the vertebrate tree based on mitochondrial small subunit rRNA is not necessarily a true tree in every detail, thus the comparison must be handled with certain cautions.

For the adenoviruses, the protease gene was chosen, as it is one of the genes available from the highest number of serotypes originating from different animal species as well as being very suitable for phylogenetic calculations (Harrach and Benkő, 1998). As a calculation method, the distance matrix analysis was selected because it had been found to provide consistent results with the different adenovirus genes (Harrach and Benkö, 1998). However, the use of hexon, DNA polymerase or pVIII amino acid or DNA sequences, and the application of parsimony analysis of these sequences all resulted in very similar distance trees (data available upon request).

Although the shape of such trees can be selected arbitrarily, the sequence of bifurcations and the branch length correspond, and are proportional, to the actual genetic distance between the representative sequences. The order of the species on the two trees is not completely identical, nevertheless the position of the classes of mammals and birds are comparable to that of the mastadeno- and aviadenoviruses (Fig. 1). The members of the proposed genus Atadenovirus ap- 
pear at a distance that corresponds to the class of reptiles or even lower level vertebrates. This would mean, that although most adenoviruses coevolved with their respective hosts, cattle, sheep, duck and fowl could have later been infected by reptilian (or other lower vertebrate) adenoviruses as well.
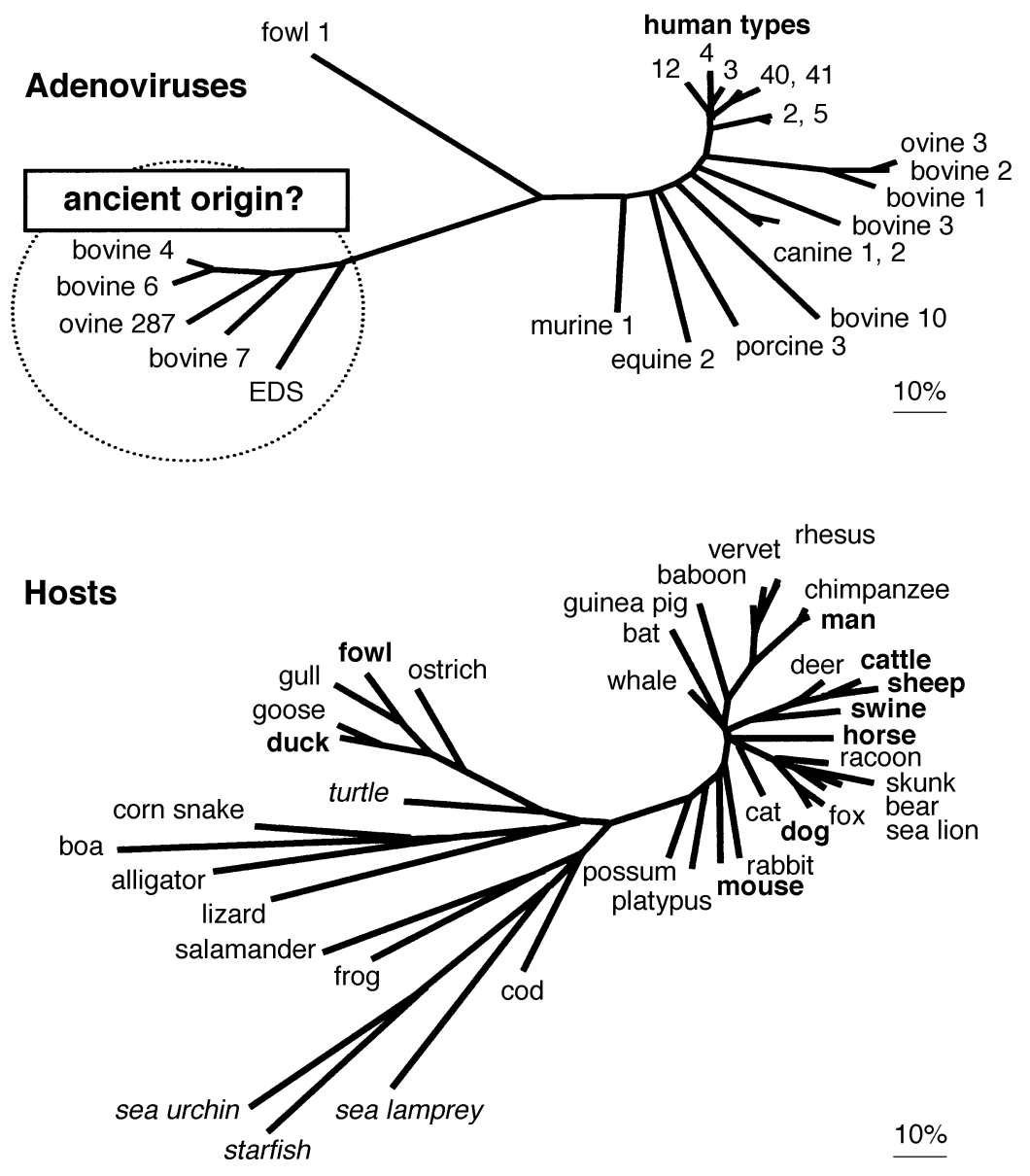

Fig. 1. The similarity of the distance trees of adenoviruses and their hosts suggests that most adenoviruses coevolved with the host while certain 'bovine', 'ovine' and 'avian' adenoviruses evolved in ancient vertebrates and were only later adapted to the present-day host. Animal name in bold: protease sequence is available from adenovirus isolated from this host; in italics: no adenovirus described. The length of the branches is proportional to the number of substitutions between any two nodes. The bar represents $10 \%$ difference between the sequences from two animal species or virus types. Note that bovine adenovirus 4, 6, and 7 are as or more distant from the mammalian and avian adenoviruses as reptiles, amphibians or bony fish are from mammals or birds 
The higher AT content observed in the chromosomal DNA of coldblooded vertebrates (Bernardi et al., 1988) further supports our hypothesis. The shorter genome length could also indicate a more ancient type of organization. It is reasonable to suppose that the structural proteins (V \& IX) and the early regions (E1A \& E3; host cell modulatory and immunomodulatory functions) missing from the avi- and atadenoviruses have been gradually acquired by the mastadenoviruses from the host cells during evolution.

The direction of adenoviral evolution may be confirmed when DNA sequences from snake or frog are available.

\section{Acknowledgement}

This work was supported by Hungarian Scientific Research Fund grants OTKA T022405 and A312.

\section{References}

Barbezange, C., Benkő, M., Dán, Á. and Harrach, B. (2000): DNA sequencing and phylogenetic analysis of the protease gene of ovine adenovirus 3 suggest that adenoviruses of sheep belong to two different genera. Virus Res. 66, 79-85.

Bartha, A. (1969): Proposal for subgrouping of bovine adenoviruses. Acta Vet. Acad. Sci. Hung. 19, 319-321.

Benkö, M. (1990): Comparison and characterization of adenoviruses of domestic Artiodactyles by means of nucleic acid analysis (in Hungarian). Ph.D. Thesis, Hungarian Academy of Sciences, Budapest.

Benkő, M., Bartha, A. and Wadell, G. (1988): DNA restriction enzyme analysis of bovine adenoviruses. Intervirol. 29, 346-350.

Benkő, M. and Harrach, B. (1998): A proposal for a new (third) genus within the family Adenoviridae. Arch. Virol. 143, 829-837.

Benkő, M., Harrach, B. and D'Halluin, J. C. (1990): Molecular cloning and physical mapping of the DNA of bovine adenovirus serotype 4: study of the DNA homology among bovine, human and porcine adenoviruses. J. Gen. Virol. 71, 465-469.

Benkő, M., Harrach, B. and Russell, W. C. (2000): Family Adenoviridae. In: Van Regenmortel, M. H. V., Fauquet, C. M., Bishop, D. H. L., Carstens, E. B., Estes, M. K., Lemon, S. M., Maniloff, J., Mayo, M. A., McGeoch, D. J., Pringle, C. R. and Wickner, R. B. (eds) Virus Taxonomy. Seventh Report of the International Committee on Taxonomy of Viruses. Academic Press, New York, San Diego, pp. 227-238.

Bernardi, G., Mouchiroud, D., Gautier, C. and Bernardi, G. (1988): Compositional patterns in vertebrate genomes: conservation and change in evolution. J. Mol. Evol. 28, 7-18.

Bloch, B., Mellergaard, S. and Nielsen, E. (1986): Adenovirus-like particles associated with epithelial hyperplasias in dab, Limanda limanda (L.). J. Fish Dis. 9, 281-285.

Boros, G., Graf, Z., Benkő, M. and Bartha, A. (1985): Isolation of a bovine adenovirus from fallow deer (Dama dama). Acta Vet. Hung. 33, 119-123.

Chiocca, S., Kurzbauer, R., Schaffner, G., Baker, A., Mautner, V. and Cotton, M. (1996): The complete DNA sequence and genomic organization of the avian adenovirus CELO. J. Virol. 70, 2939-2949.

Clark, H. F., Michalski, F., Tweedell, K. S., Yohn, D. and Zeigel, R. F. (1973): An adenovirus, FAV-1, isolated from the kidney of a frog (Rana pipiens). Virology 51, 392-400. 
Dán, Á., Ruzsics, Zs., Russell, W. C., Benkő, M. and Harrach, B. (1998): Analysis of the hexon gene sequence of bovine adenovirus type 4 provides further support for a new adenovirus genus (Atadenovirus). J. Gen. Virol. 79, 1453-1460.

Davison, A. J., Telford, E. A. R., Watson, M. S., McBride, K. and Mautner, V. (1993): The DNA sequence of adenovirus type 40. J. Mol. Biol. 234, 1308-1316.

Felsenstein, J. (1989): PHYLIP-Phylogeny inference package (version 3.2). Cladistics 5, 164-166.

Harrach, B. and Benkő, M. (1998): Phylogenetic analysis of adenovirus sequences. Proof of the necessity of establishing a third genus in the Adenoviridae family. In: Wold, W. S. M. (ed.) Adenovirus Methods and Protocols (Methods in Molecular Medicine, Vol. 21). Humana Press Inc., Totowa, N. J., USA, pp. 309-339.

Harrach, B., Meehan, B. M., Benkő, M., Adair, B. M. and Todd, D. (1997): Close phylogenetic relationship between egg drop syndrome virus, bovine adenovirus serotype 7 , and ovine adenovirus strain 287. Virology 229, 302-306.

Hedrick, R. P., Speas, J., Kent, M. L. and McDowell, T. (1985): Adenolike virus associated with a disease of cultured white sturgeon Acipenser transmontanus. Can. J. Fish. Aquat. Sci. 42, $1231-1235$

Hess, M., Blöcker, H. and Brandt, P. (1997): The complete nucleotide sequence of the egg drop syndrome virus: an intermediate between mastadenoviruses and aviadenoviruses. Virology 238, $145-156$

Horner, G. W., Hunter, R., Bartha, A. and Benkő, M. (1989): A new subgroup 2 bovine adenovirus proposed as the prototype strain 10. Arch. Virol. 109, 121-124.

Jacobson, E. R., Gardiner, C. H. and Foggin, C. M. (1984): Adenovirus-like infection in two Nile crocodiles. J. Am. Vet. Med. Assoc. 185, 1421-1422.

Jacobson, E. R., Kopit, W., Kennedy, F. A. and Funk, R. S. (1996): Coinfection of a bearded dragon, Pogona vitticeps, with adenovirus- and dependovirus-like viruses. Vet. Pathol. 33, 343-346.

Juhasz, A. and Ahne, W. (1993): Physicochemical properties and cytopathogenicity of an adenovirus-like agent isolated from corn snake (Elaphe guttata). Arch. Virol. 130, 429-439.

Kiss, I., Matiz, K., Allard, A., Wadell, G. and Benkő, M. (1996): Detection of homologous DNA sequences in animal adenoviruses by polymerase chain reaction. Acta Vet. Hung. 44, 243-251.

Lakatos, B., Farkas, J., Egberink, H. F., Vennema, H., Horzinek, M. C. and Benkő, M. (1999): Detection of persistent adenovirus infection in a cat by polymerase chain reaction. Acta Vet. Hung. 47, 493-497.

Lehmkuhl, H. D., Hobbs, L. A. and Woods, L. W. (1999): Characterization of a new adenovirus isolated from black-tailed deer in California. EMBL Nucleotide Sequence Database/ GenBank. Accession number AF198354.

Maidak, B. L., Olsen, G. J., Larsen, N., Overbeek, R., McCaughey, M. J. and Woese, C. R. (1997): The RDP (Ribosomal Database Project). Nucleic Acid Res. 25, 109-111.

Matiz, K., Ursu, K., Harrach, B., Zádori, Z. and Benkő, M. (1998): Sequencing and phylogenetic analysis of the protease gene, and genetic mapping of bovine adenovirus type 10 define its relatedness to other bovine adenoviruses. Virus Res. 55, 29-35.

Page, R. D. M. (1996): TREEVIEW: An application to display phylogenetic trees on personal computers. Computer Applications in the Biosciences 12, 357-358.

Pring-Åkerblom, P., Blazek, K., Schramlová, J. and Kunstyr, I. (1997): Polymerase chain reaction for detection of guinea pig adenovirus. J. Vet. Diagn. Invest. 9, 232-236.

Russell, W. C. and Benkő, M. (1999): Adenoviruses (Adenoviridae): animal viruses. In: Webster, R. G. and Granoff, A. (eds) Encyclopedia of Virology. Academic Press, London, pp. 14-21.

Vrati, S., Brookes, D. E., Strike, P., Khatri, A., Boyle, D. B. and Both, G. W. (1996): Unique genome arrangement of an ovine adenovirus: identification of new proteins and proteinase cleavage sites. Virology 220, 186-199.

Wigand, R., Bartha, A., Dreizin, R. S., Esche, H., Ginsberg, H. S., Green, M., Hierholzer, J. C., Kalter, S. S., McFerran, J. B., Petterson, U., Russell, W. C. and Wadell, G. (1982): Adenoviridae: second report. Intervirol. 18, 169-176. 